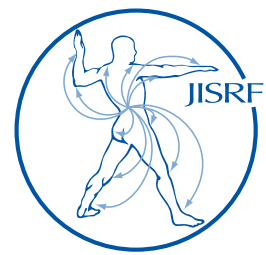

\title{
Posterior Femoral Single Limb Osteotomy for the Removal of Well-Fixed Modular Femoral Neck Components
}

\author{
Fehring $K^{1}$, Martin $R^{1}$, Sculco $P^{2}$, Kalantari $S^{2}$, Trousdale $R^{2}$
}

\begin{abstract}
Modular neck femoral components were introduced to optimize femoral neck anteversion, leg length, offset, and stability in total hip arthroplasty. However, concerns have been raised in recent years regarding early failure of these implants due to corrosion, pseudotumor, as well as fracture of the modular neck. Removing modular neck femoral implants is challenging as removal of the modular femoral neck leaves a proximally coated femoral stem level with the proximal bone of the femoral neck. We describe a posterior femoral single limb osteotomy (posterior cut of an extended trochanteric osteotomy) for the removal of a modular neck femoral component.
\end{abstract}

Keywords: femoral osteotomy; THA; revision THA

Level of Evidence: AAOS Therapeutic Level III

\section{Introduction}

Modular neck femoral components were introduced to optimize femoral neck anteversion, leg length, offset, and stability in total hip arthroplasty. [1] However, concerns have been raised in recent years regarding early failure of these implants due to corrosion, pseudotumor, as well as fracture of the modular neck. [2-7] Due to these early failure modes, the revision of these femoral components has become more common. Additionally, we have noted that implant removal of well-fixed modular femoral neck implants can be challenging secondary to the modularity of the femoral neck. Extended trochanteric osteotomies have previously been utilized for the extraction of these implants due to the difficulty with removal. []]

Extended trochanteric osteotomies (ETO) have been described for the removal of well-fixed femoral components in revision total hip arthroplasty. [ㅁ-10] While this
1 Keith A Fehring, MD; J. Ryan Martin, MD OrthoCarolina, Hip and Knee Center, 2001 Vail Avenue Suite 200A, Charlotte, NC 28207

(Direct reprint requests to Ryan Martin)

2 Peter K. Sculco, MD; Saaed Kalantari, BS; Robert T Trousdale, MD Mayo Clinic, Department of Orthopaedic Surgery, Rochester, MN 55905 
technique has proved useful in the removal of components with good results at mid-term follow up, $[\underline{8}, \underline{11}, \underline{12}]$ stem subsidence, trochanteric migration, nonunion, and fracture of the fragment have been reported. [11-13]

Removing modular neck femoral implants is challenging as removal of the modular femoral neck leaves a proximally coated femoral stem level with the proximal bone of the femoral neck. Without a femoral neck, universal extraction devices and vice grip instruments can not be used. This emphasizes the importance of having implant specific disimpaction equipment available at time of revision in addition to alternative methods for implant removal. We have identified a bone-preserving posterior femoral "single limb osteotomy" that limits the complications associated with an ETO. The purpose of this report was to describe a posterior femoral single limb osteotomy (posterior cut of an extended trochanteric osteotomy) for the removal of a modular neck femoral component.

\section{Surgical Technique}

A standard posterior approach to the hip was utilized for this technique. Pericapsular scar was then removed in order to expose the proximal femur. The hip was dislocated posteriorly and the vastus lateralis was elevated distally to expose the posterior aspect of the femur. The modular head and neck were removed with the manufacturers' standard extraction instruments (Stryker, Mawah, NJ, USA). A round tip burr was used to expose the lateral shoulder of the implant to prevent impingement on the greater trochanter during extraction. A standard pencil tip burr followed by a longer pencil tip burr was used to disrupt the bone-implant interface proximally. The standard Stryker Rejuvenate (Stryker Corporation, Mawah, NJ) stem extractor was then placed onto the proximal portion of the implant. A slap hammer was then used to attempt removal of the implant. Traditionally, if this was unsuccessful, an extended trochanteric osteotomy is performed for removal of the implant. In this case, a posteriorly based femoral single limb osteotomy was utilized to open the femur for easier extraction. A microsagittal saw was used to make this osteotomy beginning from the posterior aspect of the greater trochanter extending distally to the templated length (typically $12-14 \mathrm{~cm}$ ) and represents the posterior limb of a standard extended trochanteric osteotomy. [ㅁ-10] (Figure 1) Osteotomes were then inserted to slightly expand the femoral canal diameter. This osteotomy decreases hoop stresses around the femoral implant and may help to disrupt the bone implant interface in well fixed implants.

The stem extractor was again utilized for stem extrac-

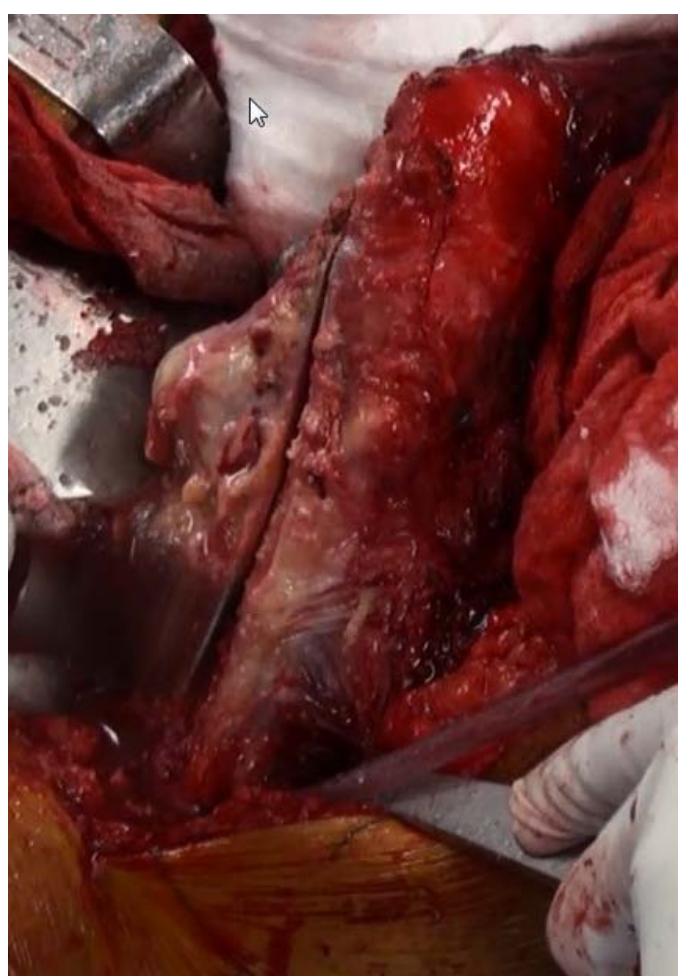

Figure 1. tion. The femoral single limb osteotomy allows the diameter of the femur to expand decreases hoop stresses facilitating implant removal. (Figures 2 and 3) At the time of closure, two 18 gauge wires are circumferentially passed around the femur and tightened to close the osteotomy. If the implant is still not easily extracted following the posterior osteotomy, the osteotomy can readily be transitioned to a standard extended trochanteric osteotomy.

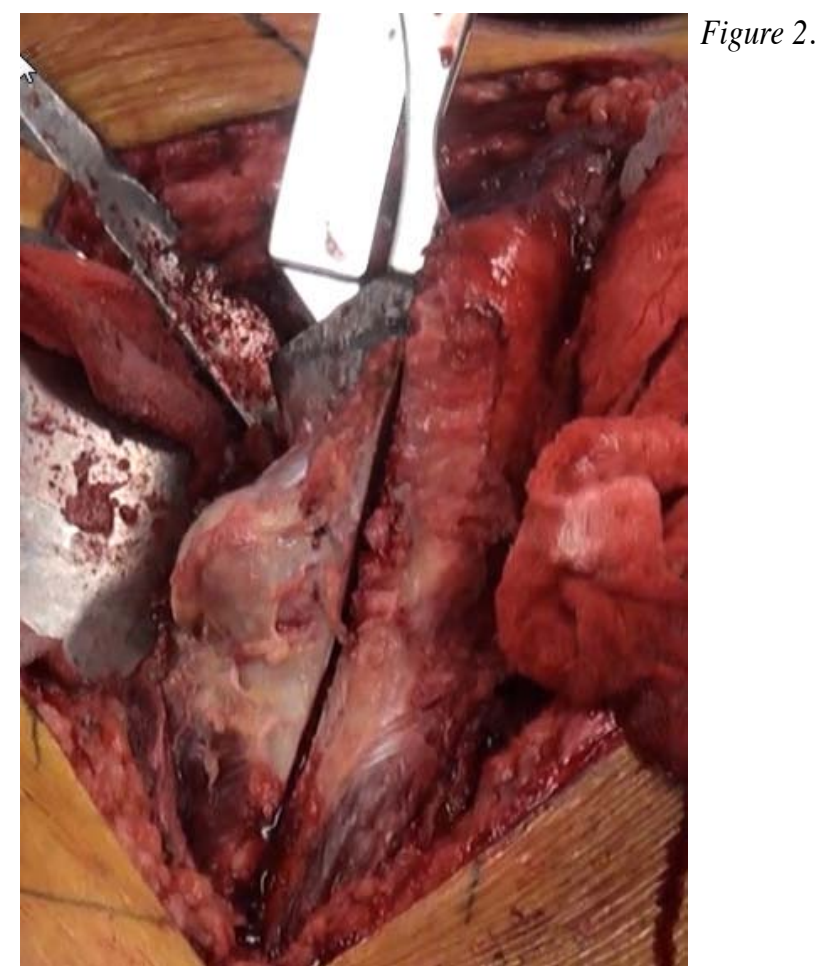




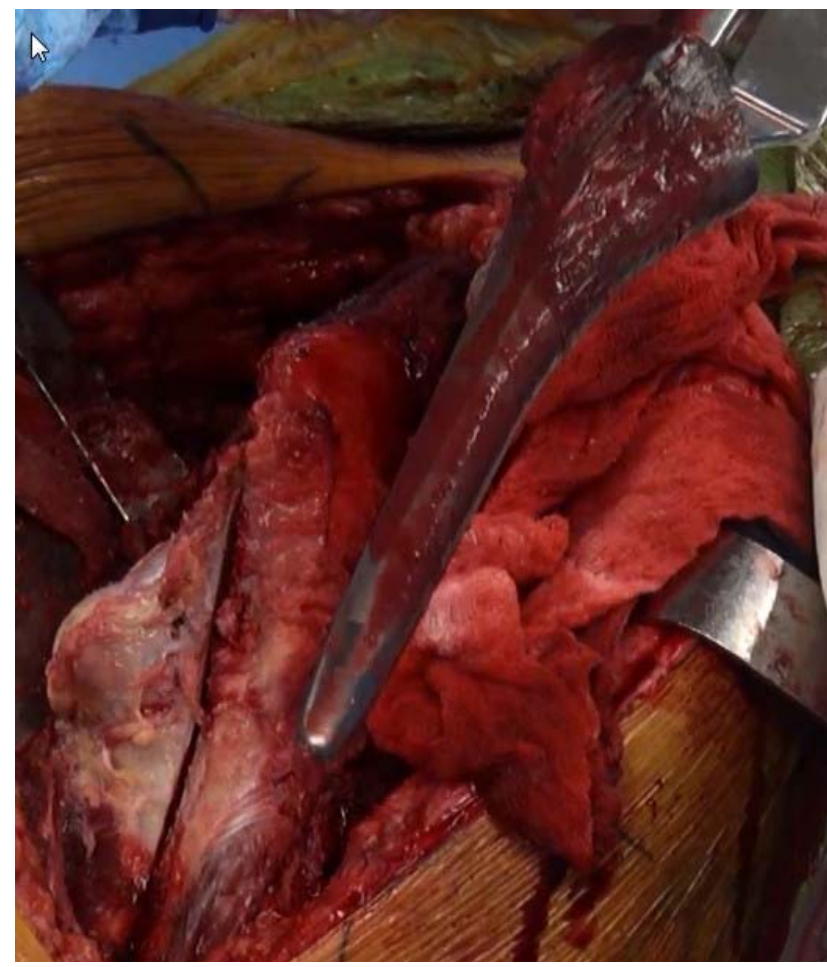

Figure 3.

\section{Case Report}

A 60-year-old male 4 years after a right total hip arthroplasty for osteoarthritis presented to our institution with a chief complaint of hip pain. Implanted components consisted of a Tritanium acetabular shell and a Rejuvenate modular neck femoral stem (Stryker, Mawah, NJ, USA). His post-operative course was complicated by a post-operative hematoma, managed non-operatively with no additional sequela. Over the last two years his hip pain had progressively worsened over, requiring a cane for ambulation. Prior work up included ESR, CRP, cobalt and chromium within normal limits and a bone scan suggestive of acetabular loosening. On physical examination he walked with a markedly antalgic gait with a positive Trendelenburg test on the right. Hip pain was reproducible with resisted flexion and internal rotation. Radiographic evaluation showed a well-fixed femoral stem and loosening of the acetabular component. (Figure 4)

On follow-up evaluation, repeat inflammatory markers were significant elevated with ESR 37 (normal 0-22) and CRP 56.8 (normal $<8.0 \mathrm{mg} / \mathrm{L}$ ). Intraarticular hip aspiration contained 3036 total nucleated cells and $85 \%$ neutrophils concerning for periprosthetic infection. At time of revision surgery, intraoperative pathology was positive for acute inflammation and a resection arthroplasty was performed. The posterior femoral single limb osteotomy was utilized for removal of the femoral component. (Video) Af-

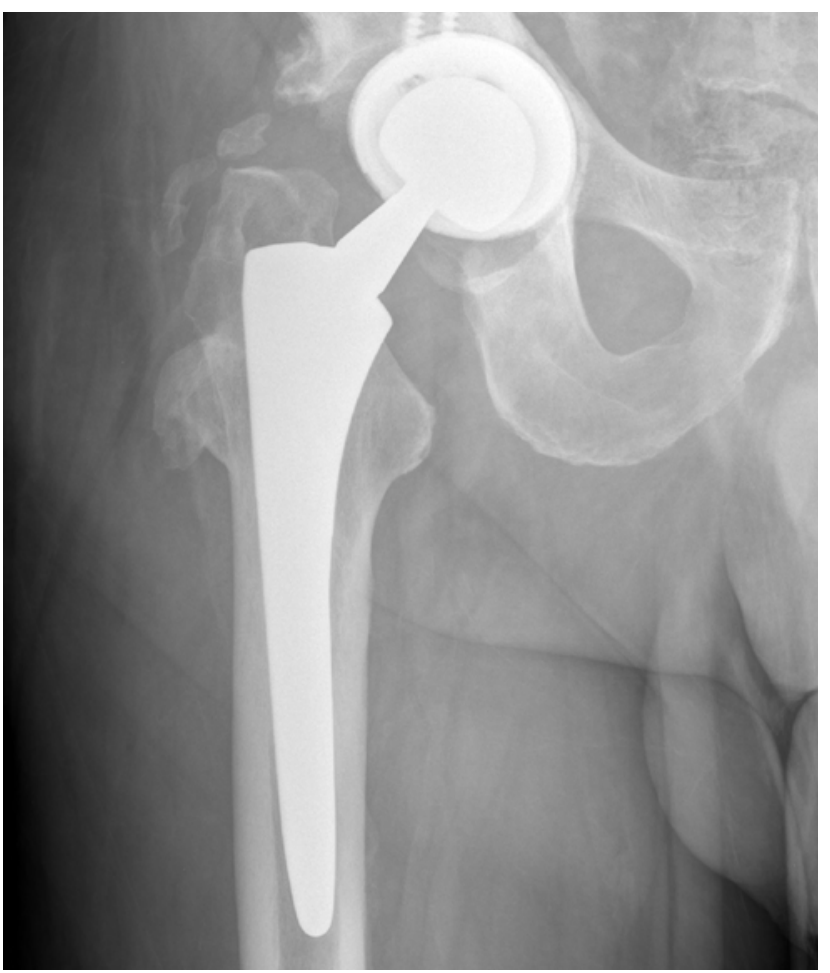

Figure 4.

ter stem removal and debridement, a temporary antibiotic articulating spacer (Prostalac, DePuy, Warsaw, IN) was placed. (Figure 5) Intraoperative cultures grew methicillin resistant staphylococcus epidermidis. Infectious Disease was consulted and the patient was placed on intravenous vancomycin for 6 weeks. Postoperatively, the patient was made partial weight bearing and was discharged from the hospital on post-operative day 4 (Figure 6).

\section{Discussion}

Modular neck femoral stem designs were introduced to address concerns associated with instability after THA. These implants gained popularity due to their ability to independently adjust leg length, offset and version through modularity of the neck segment. In recent years, several failure modes of modular

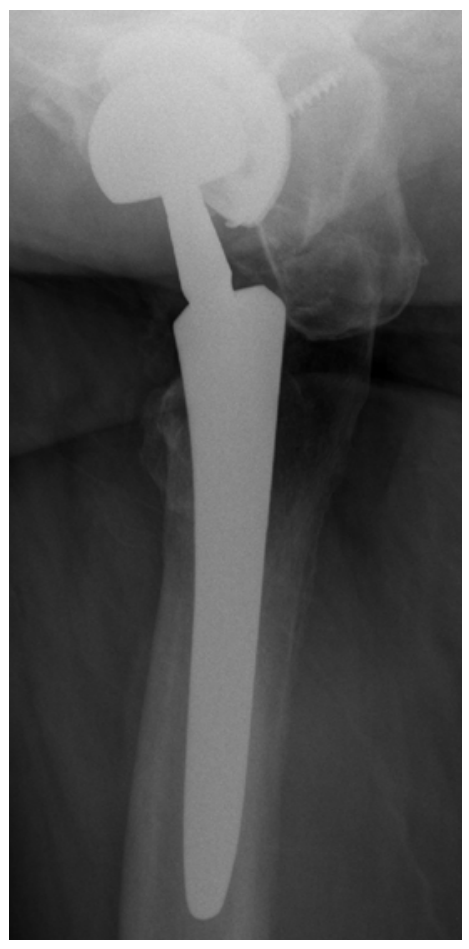

Figure 5. 
neck have been identified including modular neck fracture, corrosion, and pseudotumor $[\underline{2}, \underline{3}, \underline{6}, \underline{1}, \underline{14}]$. The corrosion products are similar to those seen with metal-on-metal bearings and trunnion wear associated with metalon-polyethylene bearings $[\underline{2}, \underline{15}]$. (I would just remove this line) Removal of these modular neck stems can be challenging as removal of the modular neck prevents the use of universal femoral disimpaction devices and an ETO has often been necessary for removal.

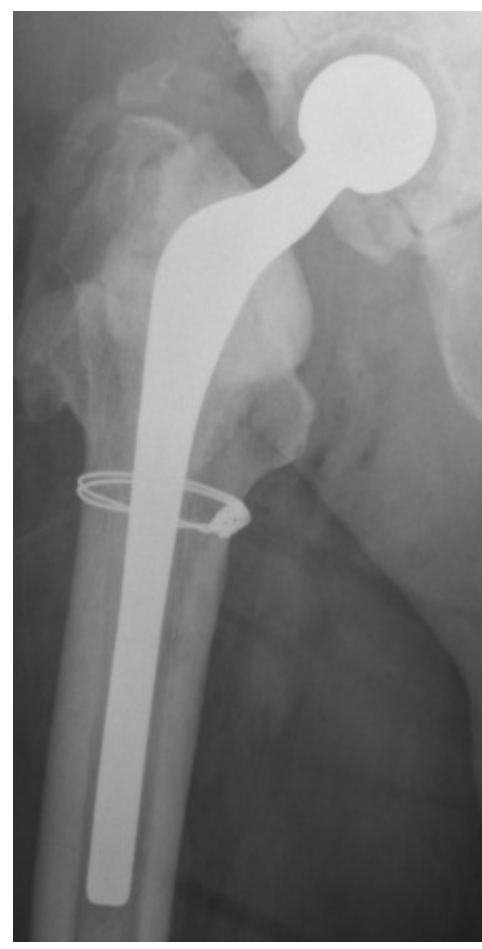

Figure 6.
[5] .The posterior single limb osteotomy described in this paper is bone and soft-tissue preserving and facilitates the extraction of a well-fixed modular femoral neck implant. The benefits of a single limb osteotomy is that it minimizes the risk of fracture of the osteotomy fragment, trochanteric migration, implant subsidence, and trochanteric nonunion associated with an ETO [ $\underline{8}, \underline{10}-\underline{13}]$. A posterior single limb osteotomy also facilitates subsequent fixation as the metaphyseal tube is still intact and does not require reduction. There is also little downside to attempting implant removal after completing the single limb as it can be easily converted to an ETO if necessary. To date, we have been able to successfully remove all modular neck implants utilizing this technique without the need for an extended trochanteric osteotomy.

Bauze et al. [16] previously described a posterior longitudinal osteotomy in the removal of 12 uncemented femoral implants. Ten of these stems had a collar making it difficult to access the medial calcar. The majority of these stems were of older designs no longer in use. The authors found that type of coating (plasma-sprayed or hydroxyapatite), the degree of coating (proximally or fully coated), and length of the stem did not affect the ability to disimpact the stem and all osteotomies healed uneventfully without complication. A single limb osteotomy has also been described for both the direct anterior and direct lateral approaches and with the difference being that the longitudinal split is anterior to that of a standard posteriorly based ETO. $[17, \underline{18}]$
In summary, the posterior femoral single limb osteotomy is an effective technique for the removal of proximal ingrowth stems, particularly modular neck femoral stems where engagement of the femoral neck for removal is not possible. There is little downside to this technique as a complete ETO can be performed if necessary. Fully porous coated implants may require a complete ETO, but the authors believe the posterior single limb osteotomy is an effective technique for the removal of all proximally coated monoblock and modular femoral stems.

\section{Disclosure}

The authors declare that there is no conflict of interest regarding the publication of this paper. For full disclosures refer to last page of this journal.

\section{References}

1. Tsai SJ, Wang CT, Jiang CC. The effect of posterior capsule repair upon post-operative hip dislocation following primary total hip arthroplasty. BMC Musculoskelet Disord. 2008;9:29-2474-9-29.

2. Suh KT, Park BG, Choi YJ. A posterior approach to primary total hip arthroplasty with soft tissue repair. Clin Orthop Relat Res. 2004;(418)(418):162-167.

3. Tippets DM, Zaryanov AV, Burke WV, et al. Incidence of heterotopic ossification in direct anterior total hip arthroplasty: A retrospective radiographic review. J Arthroplasty. 2014;29(9):1835-1838.

4. Sierra RJ, Beaule P, Zaltz I, et al. Prevention of nerve injury after periacetabular osteotomy. Clin Orthop Relat Res. 2012;470(8):2209-2219.

5. De Martino I, Triantafyllopoulos GK, Sculco PK, Sculco TP. Dual mobility cups in total hip arthroplasty. World J Orthop. 2014;5(3):180-187.

6. Nunley RM, Zhu J, Clohisy JC, Barrack RL. Aspirin decreases heterotopic ossification after hip resurfacing. Clin Orthop Relat Res. 2011;469(6):1614-1620.

7. Pellicci PM, Bostrom M, Poss R. Posterior approach to total hip replacement using enhanced posterior soft tissue repair. Clin Orthop Relat Res. 1998;(355)(355):224228.

8. Dudda M, Gueleryuez A, Gautier E, Busato A, Roeder C. Risk factors for early dislocation after total hip arthroplasty: A matched case-control study. J Orthop Surg (Hong Kong). 2010;18(2):179-183.

9. Woolson ST, Rahimtoola ZO. Risk factors for dislocation during the first 3 months after primary total hip replacement. J Arthroplasty. 1999;14(6):662-668.

10. Soong M, Rubash HE, Macaulay W. Dislocation after total hip arthroplasty. J Am Acad Orthop Surg. 2004;12(5):314-321.

11. Lee BP, Berry DJ, Harmsen WS, Sim FH. Total hip arthroplasty for the treatment of an acute fracture of the femoral neck: Long-term results. J Bone Joint Surg Am. 1998;80(1):70-75.

12. Berry DJ, von Knoch M, Schleck CD, Harmsen WS. The cumulative long-term risk of dislocation after primary charnley total hip arthroplasty. J Bone Joint Surg Am. 2004;86-A(1):9-14

13. Cohn RM, Della Valle AG, Cornell CN. Heterotopic ossification is less after THA in patients who receive aspirin compared to coumadin. Bull NYU Hosp Jt Dis. 2010;68(4):266-272

14. Oni JK, Pinero JR, Saltzman BM, Jaffe FF. Effect of a selective COX-2 inhibitor, celecoxib, on heterotopic ossification after total hip arthroplasty: A case-controlled study. Hip Int. 2014;24(3):256-262.

15. Hedley AK, Hendren DH, Mead LP. A posterior approach to the hip joint with complete posterior capsular and muscular repair. J Arthroplasty. 1990;5 Suppl:S57-66.

16. Bauze AJ, Charity J, Tsiridis E, Timperley AJ, Gie GA. Posterior longitudinal split osteotomy for femoral component extraction in revision total hip arthroplasty. J Arthroplasty. 2008;23(1):86-89.

17. Manrique J, Chen AF, Heller S, Hozack WJ. Direct anterior approach for revision total hip arthroplasty. Ann Transl Med. 2014;2(10):100-5839.2014.09.11.

18. Taylor JW, Rorabeck CH. Hip revision arthroplasty. approach to the femoral side. Clin Orthop Relat Res. 1999;(369)(369):208-222. 